also need to be able to make two qubits interact," said Schoelkopf. "With this experiment we don't just operate one gate; we string together 10 one-qubit gates and 2 two-qubit gates."

"Both qubits in the two-qubit gates have to work at the same time, so you have to be able to reliably make two qubits with long coherence times," said Steve Girvin, co-author of the article and co-principal investigator. "We used a charge-based qubit, which normally would be sensitive to electrical noise. But we developed one that stays insensitive to noise for a long time, up to three microseconds."

"There's a tension between using larger- scale manmade systems like ours as qubits, which are easier to make, test and control, versus using individual atoms, which stay coherent longer, but are much more difficult to couple together in complex ways," said Schoelkopf.

"But there's an advantage to using a superconducting circuit, which is all controlled electronically," he said. "The goal is to make a scalable device, with thousands and thousands of qubits working together. This is still a long way off, but the idea of using standard integrated circuit technology makes it easier to imagine that it might be possible someday."

Although the quantum processor itself must be kept just above absolute zero in order to maintain the superconducting properties of the circuit, DiCarlo said that the rest of the system looks like a typical processor, with only wires going into the system and wires coming out.

But Schoelkopf cautions it will still be some time before solid-state quantum computers become the industry standard.

"I'm relatively optimistic that we should be able to combine three or more qubits soon," Schoelkopf said. "But to make a system which will actually perform computations on your laptop would take a thousand qubits. It's hard to see that far into the future, but this experiment is a significant step forward."

\section{Creep in Concrete Occurs at the Nanoscale Level}

M. Vandamme of Université Paris-Est and F.-J. Ulm of the Massachusetts Institute of Technology (MIT) have determined that creep in concrete is caused by the rearrangement of particles at the nanoscale. Ulm, a professor in the Department of Civil and Environmental Engineering at MIT, said, "We can't prevent creep from happening, but if we slow the rate at which it occurs, this will increase concrete's durability and prolong the life of the structures."

Previously, Ulm had discovered that the basic building block of cement paste at the nanoscale-calcium-silicate-hydrates, or C-S-H-is granular in nature. His research had demonstrated that $\mathrm{C}-\mathrm{S}-\mathrm{H}$ naturally self-assembles at two structurally distinct but chemically similar phases when mixed with water, each with a fixed packing density close to one of the two maximum densities allowed by nature for spherical objects $(64 \%$ for the lower density and $74 \%$ for high).

Now, as reported in the Proceedings of the National Academy of Sciences online Early Edition the week of June 15 (DOI: 10.1073/pnas.0901033106), Ulm and Vandamme explain that concrete creep comes about when these nanometersized C-S-H particles rearrange into altered densities: some looser and others more tightly packed.

They also explain that a third, more dense phase of C-S-H can be induced by manipulating the cement mix with other minerals such as silica fumes, a waste material of the aluminum industry. These reacting fumes form additional smaller particles that fit into the spaces between the nano-granules of $\mathrm{C}-\mathrm{S}-\mathrm{H}$, spaces that were formerly filled with water. This has the effect of increasing the density of C-S-H to up to $87 \%$, which in turn greatly hinders the movement of the C-S-H granules over time.
The researchers show experimentally that the rate of creep is logarithmic, which means slowing creep increases durability exponentially. They demonstrate mathematically that creep can be slowed by a rate of 2.6.

Ulm said, "With this new understanding of concrete, we could produce filigree: light, elegant, strong structures that will require far less material."

An estimated $5-8 \%$ of all humangenerated atmospheric $\mathrm{CO}_{2}$ worldwide comes from the concrete industry. According to the engineers, their research may likely lead to concrete infrastructure capable of lasting hundreds of years rather than tens, which will bring enormous cost-savings and decreased concrete-related $\mathrm{CO}_{2}$ emissions. More durable concrete means that less building material and less frequent renovations will be required.

DENISE BREHM

\section{Bandgap Controlled in Bilayer Graphene}

Graphene is the two-dimensional crystalline form of carbon, whose extraordinary electron mobility and other unique features hold great promise for nanoscale electronics and photonics. But there is a catch: Graphene has no bandgap. Now Y. Zhang of the University of California at Berkeley, F. Wang of UC-Berkeley and Lawrence Berkeley National Laboratory (LBNL), and their colleagues have engineered a bandgap in bilayer graphene that can be precisely controlled at $0-250 \mathrm{meV}$.

Moreover, the research team's experiment was conducted at room tempera- ture, requiring no refrigeration of the device. Among the applications made possible by this breakthrough are new kinds of nanotransistors and-because of its narrow bandgap-nano-light-emitting diodes and other nanoscale optical devices in the infrared range.

As reported in the June 11 issue of Nature (DOI: 10.1038/nature08105; p. 820), the researchers built a two-gated bilayer device-a field-effect transistor (FET) - which is a type of transistor that controls the flow of electrons from a source to a drain with electric fields shaped by the gate electrodes. Their nano-FET used a silicon substrate as the bottom gate, with a thin insulating layer of silicon dioxide between it and the stacked graphene layers. A transparent layer of aluminum oxide lay over the graphene bilayer; on top of that was the top gate, made of platinum.

The researchers then determined the bandgap by measuring the device's optical transmission as a function of optical wavelength. They used the infrared beamline of synchrotron light from the Advanced Light Source at LBNL, focused on the graphene layers, to measure the device's optical transmission. As the researchers tuned the electrical fields by precisely varying the voltage of the gate electrodes, 
they were able to measure variations in the light absorbed by the gated graphene layers. The absorption peak in each spectrum provided a direct measurement of the bandgap at each gate voltage.

By independently manipulating the voltage of the two gates, the researchers could control the size of the bandgap and the degree of doping of the graphene bilayer. In essence, the researchers said, they created a virtual semiconductor from a material that is not inherently a semiconductor at all.

Wang said, "The electrical performance of our demonstration device is still limited, and there are many routes to improvement, for example through extra measures to purify the substrate."

Nevertheless, he said, "We've demonstrated that we can arbitrarily change the bandgap in bilayer graphene from zero to 250 milli-electron volts at room temperature, which is remarkable in itself and shows the potential of bilayer graphene for nanoelectronics. This is a narrower bandgap than common semiconductors like silicon or gallium arsenide, and it could enable new kinds of optoelectronic devices for generating, amplifying, and detecting infrared light."

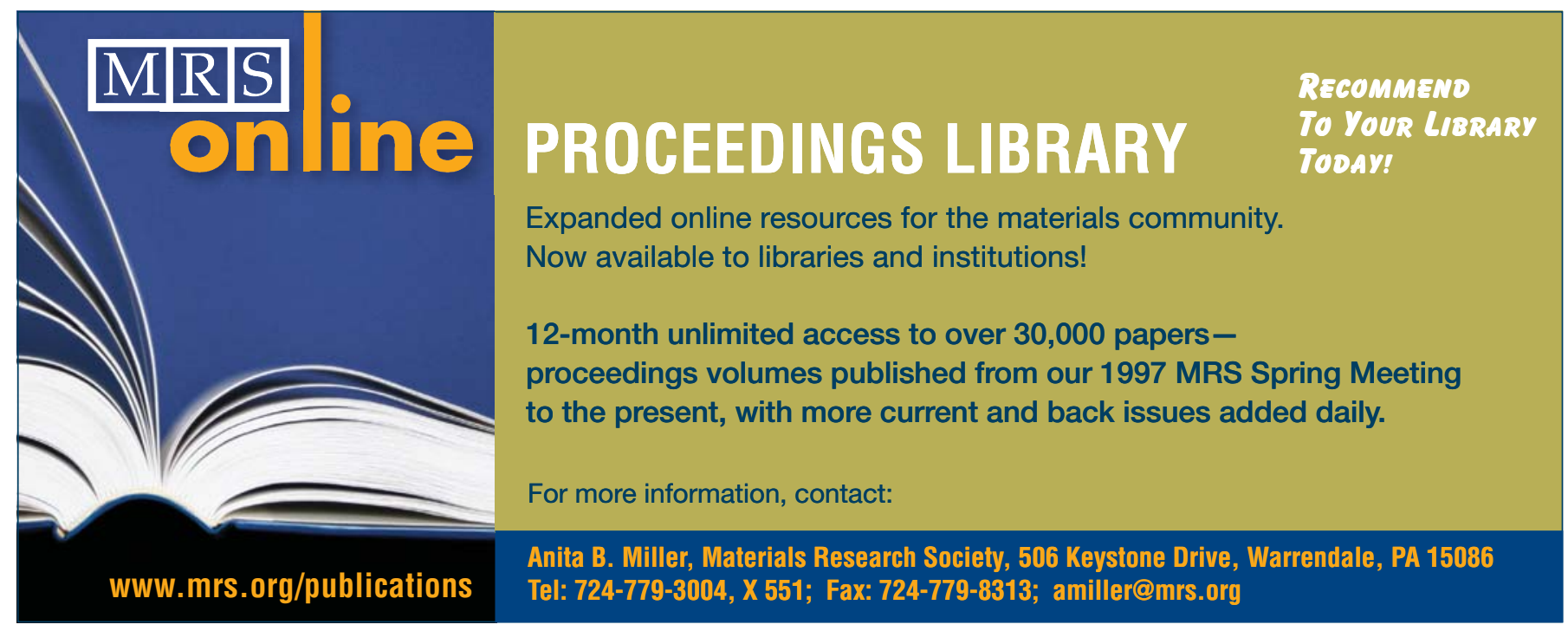

GE Global Research

There is always an exciting challenge waiting to be solved at GE Global Research. It is a place where researchers are redefining what's possible.

\section{Researchers}

The Ceramics and Metallurgy Technology (CMT) organization at GE Global Research is at the forefront of developing new and modified materials and processing techniques for exciting applications in the healthcare, water, aviation, energy and renewable power spaces. The materials and processes developed will go into some of the world's most successful and visible product offerings, impacting the world and people's lives.

We are currently looking for researchers who have unrelenting passion for technology development to join our team. Key technologies include Electronic Materials, Inorganic Optical Materials, Structural Ceramics, High temperature \& Structural alloys, Coatings, Metallurgical processes including Casting, Thermodynamics \& Kinetics, and Condensed Matter Physics.

Visit the Careers page at www.ge.com/research and search on the keywords Join CMT to view all of our current career opportunities.

imagination at work

An Equal Opportunity Employer

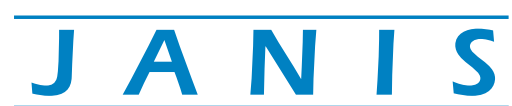

\section{Microscopy Cryostats}

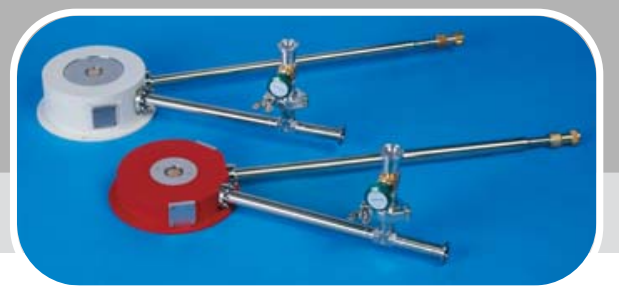

Imaging, micro-Raman, high spatial resolution photoluminescence

Ultra-low vibration $(<25 \mathrm{~nm})$

LHe models from $3.5 \mathrm{~K}-450 \mathrm{~K}$

- Compact $\mathrm{LN}_{2} / \mathrm{LHe}$ models from $10 \mathrm{~K}-450 \mathrm{~K}$

- Minimum working distance $<2 \mathrm{~mm}$

Janis Research Company

2 Jewel Drive Wilmington, MA 01887 USA

TEL +1978 657-8750 FAX +1978 658-0349 sales@janis.com

Visit our website at WWW.janis.com 